\title{
Ratio Estimator in Adaptive Cluster Sampling without Replacement of Networks
}

\author{
Nipaporn Chutiman and Monchaya Chiangpradit \\ Department of Mathematics, Faculty of Science, Mahasarakham University, Maha Sarakham 44150, Thailand \\ Correspondence should be addressed to Nipaporn Chutiman; nipaporn.c@msu.ac.th
}

Received 6 January 2014; Revised 6 May 2014; Accepted 6 May 2014; Published 27 May 2014

Academic Editor: Shein-chung Chow

Copyright (c) 2014 N. Chutiman and M. Chiangpradit. This is an open access article distributed under the Creative Commons Attribution License, which permits unrestricted use, distribution, and reproduction in any medium, provided the original work is properly cited.

\begin{abstract}
In this paper, we study the estimators of the population total in adaptive cluster sampling by using the information of the auxiliary variable. The numerical examples showed that the ratio estimator in adaptive cluster sampling without replacement of networks is more efficient than the ratio estimators in adaptive cluster sampling without replacement of units.
\end{abstract}

\section{Introduction}

Adaptive cluster sampling, proposed by Thompson [1], is an efficient method for sampling rare and hidden clustered populations. In adaptive cluster sampling, an initial sample is selected by simple random sampling without replacement of units (Thompson [1]). The unbiased estimators for adaptive cluster sampling with an initial sample taken by simple random sampling without replacement of units are HansenHurwitz and Horvitz-Thompson estimator. Although the initial sample is selected by simple random sampling without replacement of units, some networks in the sample may be selected more than once. An adaptive cluster sampling design exists with selection without replacement of networks (Salehi and Seber [2]). The unbiased estimator for adaptive cluster sampling without replacement of networks is Des Raj estimator.

The estimators mentioned above are provided by the variable of interest $(y)$ only. Sometimes other variables are related to the variable of interest. We can obtain additional information for estimating the population total. The use of an auxiliary variable is a common method to improve the precision of estimates of a population total. Dryver and Chao [3] proposed ratio estimators based on Hansen-Hurwitz and Horvitz-Thompson estimator in adaptive cluster sampling without replacement of units. In this study, we will study the estimator of population total in adaptive cluster sampling without replacement of networks using an auxiliary variable. Some comparisons are made using a simulation.

\section{Adaptive Cluster Sampling without Replacement of Units}

As in the finite population sampling situation, the population consists of $N$ units $\left(u_{1}, u_{2}, \ldots, u_{N}\right)$ indexed by their labels $(1,2, \ldots, N)$. Unit $i$ is associated as a variable of interest $y_{i}$. The vector of the population total $y$-values may be written as $\mathbf{y}=\left\{y_{1}, y_{2}, \ldots, y_{N}\right\}$. The estimator of the population total $\tau_{y}=\sum_{i=1}^{N} y_{i}$ is studied.

For adaptive cluster sampling, an initial sample of units is selected by simple random sampling without replacement. The unbiased estimator of the population total of the variable of interest is formed by using Hansen-Hurwitz estimator and Horvitz-Thompson estimator.

2.1. The Hansen-Hurwitz Estimator. The Hansen-Hurwitz estimator is based on draw-by-draw probabilities that a unit's network is intersected by the initial sample.

Let $n$ denote the initial sample size and $v$ denote the final sample size. Let $\psi_{i}$ denote the network that includes unit $i$ and let $m_{i}$ be the number of units in that network. 
The Hansen-Hurwitz estimator of the population total of the variable of interest can be written as (Thompson and Seber [4])

$$
\left(\widehat{\tau}_{y}\right)_{\mathrm{HH}}=\frac{N}{n} \sum_{i=1}^{n}\left(w_{y}\right)_{i}
$$

where $\left(w_{y}\right)_{i}$ is the average of the $y$-values in the network that includes unit $i$ of the initial sample; that is,

$$
\left(w_{y}\right)_{i}=\frac{1}{m_{i}} \sum_{j \in \psi_{i}} y_{j}
$$

The variance of $\left(\widehat{\tau}_{y}\right)_{\mathrm{HH}}$ is

$$
V\left(\widehat{\tau}_{y}\right)_{\mathrm{HH}}=\frac{N(N-n)}{n(N-1)} \sum_{i=1}^{N}\left(\left(w_{y}\right)_{i}-\frac{\tau_{y}}{N}\right)^{2} .
$$

The unbiased estimator of $V\left(\widehat{\tau}_{y}\right)_{\mathrm{HH}}$ is

$$
\widehat{V}\left(\widehat{\tau}_{y}\right)_{\mathrm{HH}}=\frac{N(N-n)}{n(n-1)} \sum_{i=1}^{n}\left(\left(w_{y}\right)_{i}-\frac{\left(\widehat{\tau}_{y}\right)_{\mathrm{HH}}}{N}\right)^{2} .
$$

2.2. The Horvitz-Thompson Estimator. The Horvitz-Thompson estimator is based on probabilities of the initial sample intersecting networks. The unbiased estimator of the population total of the variable of interest can be written as (Thompson and Seber [4])

$$
\left(\widehat{\tau}_{y}\right)_{\mathrm{HT}}=\sum_{k=1}^{K} \frac{y_{k .} I_{k}}{\alpha_{k}}=\sum_{k=1}^{\kappa} \frac{y_{k .}}{\alpha_{k}}
$$

where $y_{k}$ is the sum of $y$-values in network $k$; that is, $y_{k}$. $\sum_{i \in \psi_{k}} y_{i} . K$ is the total number of distinct networks in the population and $\kappa$ is the total number of distinct networks in the sample. $I_{k}$ is an indicator variable that takes a value of 1 with probability $\alpha_{k}$ if the initial sample intersects network $k$ and 0 otherwise. The inclusion probability of network $k$ is $\alpha_{k}=1-\left[\left(\begin{array}{c}N-m_{k} \\ n\end{array}\right) /\left(\begin{array}{l}N \\ n\end{array}\right)\right]$, and the probability that networks $j$ and $k$ are both intersected is

$$
\alpha_{j k}=1-\left\{\frac{\left.\left[\begin{array}{c}
N-m_{j} \\
n
\end{array}\right)+\left(\begin{array}{c}
N-m_{k} \\
n
\end{array}\right)-\left(\begin{array}{c}
N-m_{j}-m_{k} \\
n
\end{array}\right)\right]}{\left(\begin{array}{l}
N \\
n
\end{array}\right)}\right\} .
$$

The variance of $\left(\widehat{\tau}_{y}\right)_{\mathrm{HT}}$ is

$$
V\left(\widehat{\tau}_{y}\right)_{\mathrm{HT}}=\sum_{j=1}^{K} \sum_{k=1}^{K} \frac{y_{j .} y_{k .}\left(\alpha_{j k}-\alpha_{j} \alpha_{k}\right)}{\alpha_{j} \alpha_{k}},
$$

and the unbiased estimator of $V\left(\widehat{\tau}_{y}\right)_{\mathrm{HT}}$ is

$$
\widehat{V}\left(\widehat{\tau}_{y}\right)_{\mathrm{HT}}=\sum_{j=1}^{\kappa} \sum_{k=1}^{\kappa} \frac{y_{j .} y_{k .}}{\alpha_{j k}}\left(\frac{\alpha_{j k}}{\alpha_{j} \alpha_{k}}-1\right) .
$$

2.3. Ratio Estimators in Adaptive Cluster Sampling. Dryver and Chao [3] proposed the ratio estimator in adaptive cluster sampling based on Hansen-Horvitz's ratio estimator as

$$
\left(\widehat{\tau}_{y}\right)_{\mathrm{HH} \_\mathrm{R}}=\frac{\left(\widehat{\tau}_{y}\right)_{\mathrm{HH}}}{\left(\widehat{\tau}_{x}\right)_{\mathrm{HH}}} \tau_{x}=\widehat{R}_{\mathrm{HH}} \tau_{x}
$$

where $\widehat{R}_{\mathrm{HH}}=\left(\widehat{\tau}_{y}\right)_{\mathrm{HH}} /\left(\widehat{\tau}_{x}\right)_{\mathrm{HH}},\left(\widehat{\tau}_{x}\right)_{\mathrm{HH}}=(N / n) \sum_{i=1}^{n}\left(w_{x}\right)_{i}$, and $\left(w_{x}\right)_{i}$ is the average of the auxiliary variable $x$ in the network that includes unit $i$ of the initial sample; that is, $\left(w_{x}\right)_{i}=\left(1 / m_{i}\right) \sum_{j \in \psi_{i}} x_{j}$.

The approximated variance of $\left(\widehat{\tau}_{y}\right)_{\mathrm{HH}_{-} \mathrm{R}}$ is

$$
V\left(\widehat{\tau}_{y}\right)_{\mathrm{HH} \_\mathrm{R}}=\frac{N(N-n)}{n(N-1)} \sum_{i=1}^{N}\left(\left(w_{y}\right)_{i}-R\left(w_{x}\right)_{i}\right)^{2} .
$$

The unbiased estimator of $V\left(\widehat{\tau}_{y}\right)_{\mathrm{HH} \_\mathrm{R}}$ is

$$
\widehat{V}\left(\widehat{\tau}_{y}\right)_{\mathrm{HH} \_\mathrm{R}}=\frac{N(N-n)}{n(n-1)} \sum_{i=1}^{n}\left(\left(w_{y}\right)_{i}-\widehat{R}_{\mathrm{HH}}\left(w_{x}\right)_{i}\right)^{2} .
$$

The ratio estimator in adaptive cluster sampling based on Horvitz-Thompson's ratio estimator is

$$
\left(\widehat{\tau}_{y}\right)_{\mathrm{HT} \_\mathrm{R}}=\frac{\left(\widehat{\tau}_{y}\right)_{\mathrm{HT}}}{\left(\widehat{\tau}_{x}\right)_{\mathrm{HT}}} \tau_{x}=\widehat{R}_{\mathrm{HT}} \tau_{x}
$$

where

$$
\begin{aligned}
\widehat{R}_{\mathrm{HT}} & =\frac{\left(\widehat{\tau}_{y}\right)_{\mathrm{HT}}}{\left(\widehat{\tau}_{x}\right)_{\mathrm{HT}}} \\
\left(\widehat{\tau}_{x}\right)_{\mathrm{HT}} & =\sum_{k=1}^{\kappa}\left(\frac{x_{k .}}{\alpha_{k}}\right) .
\end{aligned}
$$

The approximated variance of $\left(\widehat{\tau}_{y}\right)_{\mathrm{HT}_{\mathrm{R}} \mathrm{R}}$ is

$$
V\left(\widehat{\tau}_{y}\right)_{\mathrm{HT} \_\mathrm{R}}=\sum_{j=1}^{K} \sum_{k=1}^{K} \frac{u_{j .} u_{k .}\left(\alpha_{j k}-\alpha_{j} \alpha_{k}\right)}{\alpha_{j} \alpha_{k}},
$$

where

$$
u_{j .}=y_{j .}-R x_{j .}
$$

The unbiased estimator of $V\left(\widehat{\tau}_{y}\right)_{\mathrm{HT} \_\mathrm{R}}$ is

$$
\widehat{V}\left(\widehat{\tau}_{y}\right)_{\mathrm{HT} \_\mathrm{R}}=\sum_{j=1}^{\kappa} \sum_{k=1}^{\kappa} \frac{u_{j .}^{\prime} u_{k \cdot}^{\prime}}{\alpha_{j k}}\left(\frac{\alpha_{j k}}{\alpha_{j} \alpha_{k}}-1\right),
$$

where

$$
u_{j .}^{\prime}=y_{j .}-\widehat{R}_{\mathrm{HT}} x_{j .} .
$$




\section{Adaptive Cluster Sampling without Replacement of Networks}

Although the initial sample is selected by simple random sampling without replacement of units, some networks in the sample may be selected more than once. An adaptive cluster sampling design exists with selection without replacement of networks (Salehi and Seber [2]).

Salehi and Seber [2] proposed a new sampling design, that is, adaptive cluster sampling with networks selected without replacement. Their procedure is as follows.

The first initial sample unit is selected by simple random sampling from the population. Then the first network is created and this network is removed from the population. Next, the second initial unit is selected by simple random sampling without replacement of the remaining units; then a second network is created. And finally, the process is continued until all of $n$ networks are selected.

Let $n$ denote the initial sample size. Let $\psi_{i}$ denote the network that includes unit $i$ and let $m_{i}$ be the number of units in that network.

Further, let $p_{i}$ be the first-draw probabilities for network which includes unit $i$. Thus $p_{i}=m_{i} / N$, where $m_{i}$ is the number of units in the network which includes unit $i$. So $p_{i} /\left(1-\sum_{j=1}^{i-1} p_{j}\right)$ is the conditional $i$ th draw probability for the $i$ th network which includes unit $i$ in the sample given the first $i-1$ networks selection.

By using a modified Des Raj estimator (Salehi and Seber [2]), an unbiased estimator for the population total of the variable of interest is

$$
\left(\widehat{\tau}_{y}\right)_{\text {Raj }}=\frac{1}{n} \sum_{i=1}^{n}\left(z_{y}\right)_{i}
$$

where, for $i=1,\left(z_{y}\right)_{i}=y_{1} / p_{1}$ and for $i=2,3, \ldots, n$ let

$$
\left(z_{y}\right)_{i}=\sum_{j=1}^{i-1} y_{j .}+\frac{\left(1-\sum_{j=1}^{i=1} p_{j}\right) y_{i}}{p_{i}} .
$$

From Raj [5] we find that $\left(z_{y}\right)_{i}$ are mutually uncorrelated so that the variance of $\left(\widehat{\tau}_{y}\right)_{\text {Raj }}$ (Salehi and Seber [2]) is

$$
V\left(\widehat{\tau}_{y}\right)_{\mathrm{Raj}}=\frac{1}{n^{2}} \sum_{i=1}^{n} V\left(z_{y}\right)_{i},
$$

and the unbiased estimator of $V\left(\widehat{\tau}_{y}\right)_{\text {Raj }}$ is

$$
\widehat{V}\left(\widehat{\tau}_{y}\right)_{\text {Raj }}=\frac{1}{n(n-1)} \sum_{i=1}^{n}\left(z_{i_{y}}-\left(\widehat{\tau}_{y}\right)_{\text {Raj }}\right)^{2} .
$$

The variance of the modified Des Raj estimator, $V\left(\widehat{\tau}_{y}\right)_{\text {Raj' }}$, is less than that of the Hansen-Hurwitz estimator, $V\left(\widehat{\tau}_{y}\right)_{\mathrm{HH}}$ (Salehi and Seber [2]).

\section{Proposed Ratio Estimator in Adaptive Cluster Sampling without Replacement of Networks}

Some other variables are related to the variable of interest. We can obtain additional information for estimating the population total. The use of an auxiliary variable is a common method to improve the precision of estimates of a population total. In this section, proposed ratio estimator in adaptive cluster sampling without replacement of networks will be described. is

The proposed ratio estimator based on Des Raj's estimator

$$
\left(\widehat{\tau}_{y}\right)_{\text {Raj } \_\mathrm{R}}=\frac{\left(\widehat{\tau}_{y}\right)_{\mathrm{Raj}}}{\left(\widehat{\tau}_{x}\right)_{\mathrm{Raj}}} \tau_{x}=\widehat{R}_{\mathrm{Raj}} \tau_{x},
$$

where $\widehat{R}_{\mathrm{Raj}}=\left(\widehat{\tau}_{y}\right)_{\mathrm{Raj}} /\left(\widehat{\tau}_{x}\right)_{\mathrm{Raj}}$ and $\left(\widehat{\tau}_{x}\right)_{\mathrm{Raj}}$ is the modified Des Raj estimator for $\tau_{x}$.

To obtain the variance of $\left(\widehat{\tau}_{y}\right)_{\text {Raj_R }}$, we write

$$
\widehat{R}_{\mathrm{Raj}}-R=\frac{\left(\widehat{\tau}_{y}\right)_{\mathrm{Raj}}}{\left(\widehat{\tau}_{x}\right)_{\mathrm{Raj}}}-R=\frac{\left(\hat{\tau}_{y}\right)_{\mathrm{Raj}}-R\left(\widehat{\tau}_{x}\right)_{\mathrm{Raj}}}{\left(\widehat{\tau}_{x}\right)_{\mathrm{Raj}}},
$$

where $R=\tau_{y} / \tau_{x}$ is the population ratio.

If $n$ is reasonably large, $\left(\widehat{\tau}_{x}\right)_{\text {Raj }}$ is closed to $\tau_{x}$ and $E\left(\widehat{R}_{\mathrm{Raj}}\right)$ is approximately equal to $R$; therefore,

$$
\begin{gathered}
\widehat{R}_{\text {Raj }}-R \approx \frac{\left(\widehat{\tau}_{y}\right)_{\text {Raj }}-R\left(\widehat{\tau}_{x}\right)_{\text {Raj }}}{\tau_{x}}, \\
V\left(\widehat{R}_{\text {Raj }}\right)=E\left[\widehat{R}_{\text {Raj }}-E\left(\widehat{R}_{\text {Raj }}\right)\right]^{2} \approx E\left[\widehat{R}_{\text {Raj }}-R\right]^{2} \\
=E\left[\frac{\left(\widehat{\tau}_{y}\right)_{\text {Raj }}-R\left(\widehat{\tau}_{x}\right)_{\text {Raj }}}{\tau_{x}}\right]^{2} \\
=\frac{1}{\tau_{x}^{2}} E\left[\left(\widehat{\tau}_{y}\right)_{\text {Raj }}-R\left(\widehat{\tau}_{x}\right)_{\text {Raj }}\right]^{2} .
\end{gathered}
$$

Let

$$
\begin{gathered}
d_{i}=\left(z_{y}\right)_{i}-R\left(z_{x}\right)_{i}, \\
\frac{1}{n} \sum_{i=1}^{n} d_{i}=\frac{1}{n} \sum_{i=1}^{n}\left(z_{y}\right)_{i}-R \frac{1}{n} \sum_{i=1}^{n}\left(z_{x}\right)_{i}=\left(\widehat{\tau}_{y}\right)_{\mathrm{Raj}}-R\left(\widehat{\tau}_{x}\right)_{\mathrm{Raj}} .
\end{gathered}
$$

So,

$$
V\left(\widehat{R}_{\mathrm{Raj}}\right)=\frac{1}{\tau_{x}^{2}} E\left(\frac{1}{n} \sum_{i=1}^{n} d_{i}\right)^{2}
$$

$$
\begin{aligned}
V\left[\frac{1}{n} \sum_{i=1}^{n} d_{i}\right] & =E\left(\frac{1}{n} \sum_{i=1}^{n} d_{i}\right)^{2}-\left[E\left(\frac{1}{n} \sum_{i=1}^{n} d_{i}\right)\right]^{2} \\
= & E\left(\frac{1}{n} \sum_{i=1}^{n} d_{i}\right)^{2}, \\
V\left(\widehat{R}_{\text {Raj }}\right) & =\frac{1}{\tau_{x}^{2}} \times V\left[\frac{1}{n} \sum_{i=1}^{n} d_{i}\right] .
\end{aligned}
$$


TABLE 1: The estimated MSE of the Hansen-Horvitz estimators and Horvitz-Thompson estimators for the population total of blue-winged teal.

\begin{tabular}{lccccc}
\hline$n$ & $E(\nu)$ & $M \widehat{S} E\left(\widehat{\tau}_{y}\right)_{\mathrm{HH}}$ & $M \widehat{S} E\left(\widehat{\tau}_{y}\right)_{\mathrm{HT}}$ & $M \widehat{S} E\left(\widehat{\tau}_{y}\right)_{\mathrm{HH} \_\mathrm{R}}$ & $M \widehat{S} E\left(\widehat{\tau}_{y}\right)_{\mathrm{HT} \_\mathrm{R}}$ \\
\hline 5 & 17.09 & $2,359,716,325$ & $1,855,257,461$ & $444,212,079$ & $444,211,995$ \\
8 & 23.44 & $1,369,069,022$ & $814,459,237$ & $155,321,999$ & $155,321,710$ \\
10 & 26.14 & $1,076,080,659$ & $527,029,260$ & $79,499,876$ & $79,499,398$ \\
15 & 31.27 & $575,418,109$ & $163,370,814$ & $11,895,009$ & $11,894,258$ \\
20 & 34.71 & $348,333,275$ & $42,718,623$ & $1,512,773$ & $1,511,986$ \\
\hline
\end{tabular}

TABLE 2: The estimated MSE of the modified Des Raj estimators for the population total of blue-winged teal.

\begin{tabular}{lccc}
\hline$n$ & $E(\nu)$ & $M \widehat{S} E\left(\widehat{\tau}_{y}\right)_{\text {Raj }}$ & $M \widehat{S} E\left(\widehat{\tau}_{y}\right)_{\text {Raj_R }}$ \\
\hline 5 & 19.90 & $1,897,377,719$ & $22,982,048$ \\
8 & 26.66 & $931,732,897$ & $15,329,005$ \\
10 & 29.84 & $654,226,056$ & $11,162,171$ \\
15 & 35.74 & $305,945,821$ & $3,298,687$ \\
20 & 39.32 & $171,618,612$ & 624,795 \\
\hline
\end{tabular}

\begin{tabular}{|c|c:c:c|c|c:c|c|c|c|}
\hline 0 & 0 & 3 & 5 & 0 & 0 & 0 & 0 & 0 & 0 \\
\hdashline 0 & 0 & 0 & 24 & 14 & 0 & 0 & 10 & 103 & 0 \\
\hdashline 0 & 0 & 0 & 0 & 2 & 3 & 2 & 0 & 13639 & 1 \\
0 & 0 & 0 & 0 & 0 & 0 & 0 & 0 & 14 & 122 \\
\hdashline 0 & 0 & 0 & 0 & 0 & 0 & 2 & 0 & 0 & 177 \\
\hline
\end{tabular}

Figure 1: Blue-winged teal data ( $y$-values).

Therefore,

$$
V\left(\left(\widehat{\tau}_{y}\right)_{\text {Raj-R }}\right)=V\left(\widehat{R}_{\text {Raj }} \tau_{x}\right)=\tau_{x}^{2} V\left(\widehat{R}_{\text {Raj }}\right) .
$$

The approximated variance of $\left(\widehat{\tau}_{y}\right)_{\text {Raj_R }}$ is

$$
V\left(\widehat{\tau}_{y}\right)_{\mathrm{Raj} \mathrm{R}}=\frac{1}{n^{2}}\left[\frac{1}{n^{2}} \sum_{i=1}^{n} V\left(d_{i}\right)\right]
$$

and the estimator of $V\left(\widehat{\tau}_{y}\right)_{\text {Raj-R }}$ is

$$
\widehat{V}\left(\widehat{\tau}_{y}\right)_{\text {Raj_R }}=\frac{1}{n^{2}}\left[\frac{1}{n(n-1)} \sum_{i=1}^{n}\left(\left(z_{y}\right)_{i}-\widehat{R}_{\text {Raj }}\left(z_{x}\right)_{i}\right)^{2}\right] .
$$

\section{Examples}

5.1. The Blue-Winged Teal Example. As an example, the bluewinged teal data (Smith et al. [6]) were used in this study. The population region of $5,000 \mathrm{~km}^{2}$ in an area of Central Florida was partitioned into fifty units of $100 \mathrm{~km}^{2}$. These data were set to be $y$-values. The total of $y$-values, $\tau_{y}$, is 14,121 (see Figure 1).

The $x$-values were simulation results from the model $x_{i}=$ $4 y_{i}-\varepsilon_{i}$, where $\varepsilon_{i} \sim N\left(0, y_{i}\right)$ (see Figure 2).

For each iteration, an initial sample of units is selected by simple random sampling without replacement. The $y$-values are obtained for keeping the sample network. In each of the

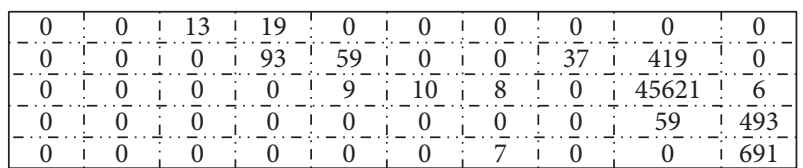

Figure 2: Simulated data ( $x$-values) from the model $x_{i}=4 y_{i}-\varepsilon_{i}$.

sample networks, the $x$-values are obtained. The condition for added units in the sample is defined by $C=\{y: y>0\}$.

For each estimator, 20,000 iterations were performed to obtain an accuracy estimate. Initial SRS sizes were varied with $n=5,8,10,15$, and 20 . The estimated variance of the estimate total is

$$
\operatorname{MSE}\left(\widehat{\tau}_{y}\right)=\frac{1}{20,000} \sum_{i=1}^{20,000}\left(\left(\widehat{\tau}_{y}\right)_{i}-\tau_{y}\right)^{2},
$$

where $\left(\widehat{\tau}_{y}\right)_{i}$ is the value for the relevant estimator for sample $i$.

For the blue-winged teal example, the estimated MSE of estimators under the adaptive cluster sampling without replacement of unit were calculated and listed in Table 1 . The estimated MSE of estimators under adaptive cluster sampling without replacement of networks were shown in Table 2.

5.2. Simulation Example. As a second illustration, we have used the simulation $x$-values and $y$-values from Chutiman and Kumphon [7] were studied. The populations were shown in Figures 3 and 4.

For each iteration, an initial sample of units is selected by simple random sampling without replacement. The $y$-values are obtained for keeping the sample network. In each sample network, the $x$-values are obtained. The condition for added units in the sample is defined by $C=\{y: y>0\}$.

For each estimator, 20,000 iterations were performed to obtain an accuracy estimate. Initial SRS sizes were varied $n=$ $5,10,15,20,30,40$, and 50 .

For the simulation example, the estimated MSE of estimators under the adaptive cluster sampling without replacement of unit were calculated and listed in Table 3 . Table 4 shows the estimated MSE of estimators under adaptive cluster sampling without replacement of networks.

From the examples, we see that the estimated MSE of adaptive cluster sampling without replacement of networks is smaller than the estimated MSE of adaptive cluster sampling without replacement of units; that is,

$$
M \widehat{S} E\left(\widehat{\tau}_{y}\right)_{\mathrm{Raj}} \leq M \widehat{S} E\left(\widehat{\tau}_{y}\right)_{\mathrm{HT}} \leq M \widehat{S} E\left(\widehat{\tau}_{y}\right)_{\mathrm{HH}} \cdot
$$


TABLE 3: The estimated MSE of the Hansen-Horvitz estimator and Horvitz-Thompson estimator for the population total of the variable of interest.

\begin{tabular}{lccccc}
\hline$n$ & $E(\nu)$ & $M \widehat{S} E\left(\widehat{\tau}_{y}\right)_{\mathrm{HH}}$ & $M \widehat{S} E\left(\widehat{\tau}_{y}\right)_{\mathrm{HT}}$ & $M \widehat{S} E\left(\widehat{\tau}_{y}\right)_{\mathrm{HH} . \mathrm{R}}$ & $M \widehat{S} E\left(\widehat{\tau}_{y}\right)_{\mathrm{HT} \cdot \mathrm{R}}$ \\
\hline 5 & 28.999 & $407,050.393$ & $398,429.963$ & $113,906.753$ & $113,871.977$ \\
10 & 50.596 & $197,774.349$ & $187,902.766$ & $55,691.349$ & $55,554.041$ \\
15 & 68.027 & $130,815.296$ & $120,108.018$ & $28,580.328$ & $28,341.920$ \\
20 & 82.526 & $95,153.494$ & $84,773.793$ & $15,778.559$ & $15,465.085$ \\
30 & 104.183 & $61,714.960$ & $52,527.501$ & $7,242.755$ & $6,890.563$ \\
40 & 120.132 & $45,383.260$ & $36,945.984$ & $5,263.785$ & $4,962.297$ \\
50 & 133.058 & $33,434.958$ & $25,939.191$ & $4,451.101$ & $4,167.722$ \\
\hline
\end{tabular}

\begin{tabular}{|c|c|c|c|c|c|c|c|c|c|c|c|c|c|c|c|c|c|c|c|}
\hline 0 & 0 & 0 & 0 & 0 & 0 & 0 & 0 & 0 & 0 & 0 & 0 & 0 & 0 & 0 & 0 & 0 & 0 & 0 & 0 \\
\hline 0 & 0 & 0 & 0 & 0 & 0 & 0 & 5 & 0 & 0 & 0 & 0 & 0 & 0 & 0 & 0 & 0 & 0 & 0 & 0 \\
\hline 0 & 0 & 0 & 0 & 0 & 0 & 2 & 22 & 5 & 0 & 0 & 0 & 0 & 0 & 0 & 0 & 0 & 0 & 0 & 0 \\
\hline 0 & 0 & 1 & 22 & 5 & 4 & 5 & 0 & 0 & 0 & 0 & 0 & 0 & 0 & 0 & 0 & 0 & 0 & 0 & 0 \\
\hline 0 & 0 & 0 & 0 & 2 & 0 & 4 & 8 & 0 & 0 & 0 & 0 & 0 & 33 & 0 & 0 & 0 & 27 & 0 & 0 \\
\hline 0 & 0 & 0 & 0 & 0 & 0 & 0 & 0 & 0 & 0 & 0 & 0 & 7 & 6 & 7 & 1 & 0 & 5 & 0 & 0 \\
\hline 0 & 0 & 0 & 0 & 0 & 0 & 0 & 21 & 0 & 0 & 1 & 0 & 0 & 0 & 0 & 0 & 0 & 0 & 0 & 0 \\
\hline 0 & 0 & 0 & 0 & 0 & 0 & 0 & 4 & 0 & 5 & 7 & 0 & 0 & 0 & 0 & 0 & 0 & 0 & 0 & 0 \\
\hline 0 & 0 & 0 & 0 & 1 & 0 & 0 & 5 & 7 & 0 & 7 & 7 & 6 & 3 & 0 & 0 & 0 & 0 & 0 & 0 \\
\hline 0 & 0 & 0 & 5 & 4 & 3 & 0 & 5 & 8 & 4 & 5 & 1 & 0 & 5 & 0 & 0 & 0 & 0 & 0 & 0 \\
\hline 0 & 7 & 65 & 0 & 4 & 5 & 0 & 9 & 0 & 0 & 0 & 0 & 0 & 3 & 1 & 0 & 0 & 0 & 0 & 0 \\
\hline 0 & 1 & 4 & 5 & 0 & 7 & 3 & 3 & 0 & 0 & 0 & 0 & 0 & 0 & 0 & 0 & 0 & 0 & 0 & 0 \\
\hline 0 & 1 & 7 & 0 & 0 & 0 & 0 & 0 & 0 & 0 & 0 & 0 & 0 & 0 & 0 & 0 & 0 & 0 & 0 & 0 \\
\hline 0 & 0 & 0 & 0 & 0 & 0 & 0 & 0 & 0 & 0 & 0 & 0 & 0 & 0 & 0 & 0 & 0 & 0 & 0 & 0 \\
\hline 0 & 0 & 0 & 0 & 0 & 0 & 0 & 0 & 0 & 0 & 0 & 0 & 0 & 0 & 0 & 0 & 0 & 0 & 0 & 0 \\
\hline 0 & 0 & 0 & 0 & 0 & 0 & 0 & 0 & 0 & 0 & 0 & 0 & 0 & 0 & 0 & 0 & 0 & 0 & 0 & 0 \\
\hline 0 & 0 & 0 & 0 & 0 & 0 & 0 & 0 & 0 & 0 & 0 & 0 & 0 & 0 & 0 & 0 & 0 & 0 & 0 & 0 \\
\hline 0 & 0 & 0 & 0 & 0 & 0 & 0 & 0 & 0 & 0 & 0 & 0 & 0 & 0 & 0 & 0 & 27 & 0 & 0 & 21 \\
\hline 0 & 0 & 0 & 0 & 0 & 0 & 0 & 0 & 0 & 0 & 0 & 0 & 0 & 29 & 0 & 0 & 0 & 0 & 0 & 0 \\
\hline 0 & 0 & 0 & 0 & 0 & 0 & 0 & 0 & 0 & 0 & 0 & 0 & 0 & 0 & 0 & 0 & 0 & 0 & 0 & 0 \\
\hline
\end{tabular}

Figure 3: The $y$-values.

\begin{tabular}{|c|c|c|c|c|c|c|c|c|c|c|c|c|c|c|c|c|c|c|c|}
\hline 0 & 0 & 0 & 0 & 0 & 0 & 0 & 0 & 0 & 0 & 0 & 0 & 0 & 0 & 0 & 0 & 0 & 0 & 0 & 0 \\
\hline 0 & 0 & 0 & 0 & 0 & 0 & 0 & 2 & 0 & 0 & 0 & 0 & 0 & 0 & 0 & 0 & 0 & 0 & 0 & 0 \\
\hline 0 & 0 & 0 & 0 & 0 & 0 & 1 & 11 & 3 & 0 & 0 & 0 & 0 & 0 & 0 & 0 & 0 & 0 & 0 & 0 \\
\hline 0 & 0 & 0 & 11 & 2 & 2 & 1 & 0 & 0 & 0 & 0 & 0 & 0 & 0 & 0 & 0 & 0 & 0 & 0 & 0 \\
\hline 0 & 0 & 0 & 0 & 1 & 0 & 2 & 4 & 0 & 0 & 0 & 0 & 0 & 12 & 0 & 0 & 0 & 15 & 0 & 0 \\
\hline 0 & 0 & 0 & 0 & 0 & 0 & 0 & 0 & 0 & 0 & 0 & 0 & 3 & 2 & 3 & 0 & 0 & 2 & 0 & 0 \\
\hline 0 & 0 & 0 & 0 & 0 & 0 & 0 & 16 & 0 & 0 & 0 & 0 & 0 & 0 & 0 & 0 & 0 & 0 & 0 & 0 \\
\hline 0 & 0 & 0 & 0 & 0 & 0 & 0 & 2 & 0 & 2 & 3 & 0 & 0 & 0 & 0 & 0 & 0 & 0 & 0 & 0 \\
\hline 0 & 0 & 0 & 0 & 0 & 0 & 0 & 2 & 2 & 0 & 3 & 3 & 2 & 1 & 0 & 0 & 0 & 0 & 0 & 0 \\
\hline 0 & 0 & 0 & 2 & 2 & 1 & 0 & 2 & 3 & 2 & 2 & 0 & 0 & 2 & 0 & 0 & 0 & 0 & 0 & 0 \\
\hline 0 & 3 & 18 & 0 & 2 & 2 & 0 & 4 & 0 & 0 & 0 & 0 & 0 & 1 & 0 & 0 & 0 & 0 & 0 & 0 \\
\hline 0 & 0 & 2 & 2 & 0 & 3 & 1 & 1 & 0 & 0 & 0 & 0 & 0 & 0 & 0 & 0 & 0 & 0 & 0 & 0 \\
\hline 0 & 0 & 3 & 0 & 0 & 0 & 0 & 0 & 0 & 0 & 0 & 0 & 0 & 0 & 0 & 0 & 0 & 0 & 0 & 0 \\
\hline 0 & 0 & 0 & 0 & 0 & 0 & 0 & 0 & 0 & 0 & 0 & 0 & 0 & 0 & 0 & 0 & 0 & 0 & 0 & 0 \\
\hline 0 & 0 & 0 & 0 & 0 & 0 & 0 & 0 & 0 & 0 & 0 & 0 & 0 & 0 & 0 & 0 & 0 & 0 & 0 & 0 \\
\hline 0 & 0 & 0 & 0 & 0 & 0 & 0 & 0 & 0 & 0 & 0 & 0 & 0 & 0 & 0 & 0 & 0 & 0 & 0 & 0 \\
\hline 0 & 0 & 0 & 0 & 0 & 0 & 0 & 0 & 0 & 0 & 0 & 0 & 0 & 0 & 0 & 0 & 0 & 0 & 0 & 0 \\
\hline 0 & 0 & 0 & 0 & 0 & 0 & 0 & 0 & 0 & 0 & 0 & 0 & 0 & 0 & 0 & 0 & 12 & 0 & 0 & 12 \\
\hline 0 & 0 & 0 & 0 & 0 & 0 & 0 & 0 & 0 & 0 & 0 & 0 & 0 & 27 & 0 & 0 & 0 & 0 & 0 & 0 \\
\hline 0 & 0 & 0 & 0 & 0 & 0 & 0 & 0 & 0 & 0 & 0 & 0 & 0 & 0 & 0 & 0 & 0 & 0 & 0 & 0 \\
\hline
\end{tabular}

FIgURE 4: The $x$-values. 
TABLE 4: The estimated MSE of the modified Des Raj estimators for the population total of the variable of interest.

\begin{tabular}{lccc}
\hline$n$ & $E(\nu)$ & $M \widehat{S} E\left(\widehat{\tau}_{y}\right)_{\text {Raj }}$ & $M \widehat{S} E\left(\widehat{\tau}_{y}\right)_{\text {Raj-R }}$ \\
\hline 5 & 31.590 & $395,296.216$ & $8,146.702$ \\
10 & 56.182 & $181,264.543$ & $7,641.379$ \\
15 & 75.244 & $116,781.850$ & $7,033.291$ \\
20 & 91.807 & $83,718.824$ & $6,417.127$ \\
30 & 116.093 & $51,340.406$ & $5,359.412$ \\
40 & 133.820 & $35,017.241$ & $4,606.211$ \\
50 & 148.388 & $25,772.744$ & $4,041.559$ \\
\hline
\end{tabular}

For the population total in adaptive cluster sampling by using the information of the auxiliary variable, we see that the estimated MSE of the ratio estimator in adaptive cluster sampling without replacement of networks is smaller than the estimated MSE of the ratio estimator in adaptive cluster sampling without replacement of units; that is,

$$
M \widehat{S} E\left(\widehat{\tau}_{y}\right)_{\mathrm{Raj}-\mathrm{R}} \leq M \widehat{S} E\left(\widehat{\tau}_{y}\right)_{\mathrm{HT} \_\mathrm{R}} \leq M \widehat{S} E\left(\widehat{\tau}_{y}\right)_{\mathrm{HH} \_\mathrm{R}} .
$$

\section{Conclusions}

We discuss using the auxiliary information, that is, the ratio estimators in adaptive cluster sampling without replacement of units and without replacement of networks. For the adaptive cluster sampling without replacement of units, HorvitzThompson's ratio estimator is better than Hansen-Hurwitz's ratio estimator (Dryver and Chao [3]).

Although the initial units in adaptive cluster sampling are selected by simple random sampling without replacement, networks may be selected more than once. So the adaptive cluster sampling without replacement of units is equivalent to the adaptive cluster sampling without replacement of networks. Therefore, the modified Des Raj ratio estimator is better than both Hansen-Hurwitz's ratio estimator and Horvitz-Thompson's ratio estimator.

\section{Conflict of Interests}

The authors declare that there is no conflict of interests regarding the publishing of this paper.

\section{Acknowledgments}

The authors would like to profoundly thank Paveen Chutiman for his programming advice. They would also like to express their special thanks to Assistant Professor Siriluck Jemjitpornchai and Prasert Vangsantitrakul for their valuable comments and suggestions on the material of this paper and for their help in correcting English as well.

\section{References}

[1] S. K. Thompson, "Adaptive cluster sampling," Journal of the American Statistical Association, vol. 85, no. 412, pp. 1050-1059, 1990.
[2] M. M. Salehi and G. A. F. Seber, "Adaptive cluster sampling with networks selected without replacement," Biometrika, vol. 84, no. 1, pp. 209-219, 1997.

[3] A. L. Dryver and C. T. Chao, "Ratio estimators in adaptive cluster sampling," Environmetrics, vol. 18, no. 6, pp. 607-620, 2007.

[4] S. K. Thompson and G. A. F. Seber, Adaptive Sampling, Wiley Series in Probability and Statistics: Probability and Statistics, John Wiley \& Sons, New York, NY, USA, 1996.

[5] D. Raj, "Some estimators in sampling with varying probabilities without replacement," Journal of the American Statistical Association, vol. 51, pp. 269-284, 1956.

[6] D. R. Smith, M. J. Conroy, and D. H. Brakhage, "Efficiency of adaptive cluster sampling for estimating density of wintering waterfowl," Biometrics, vol. 51, no. 2, pp. 777-788, 1995.

[7] N. Chutiman and B. Kumphon, "Ratio estimator using two auxiliary variables for adaptive cluster sampling," Journal of the Thai Statistical Association, vol. 6, no. 2, pp. 241-256, 2008. 


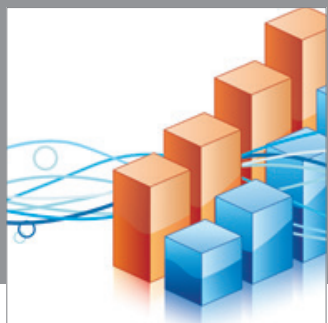

Advances in

Operations Research

mansans

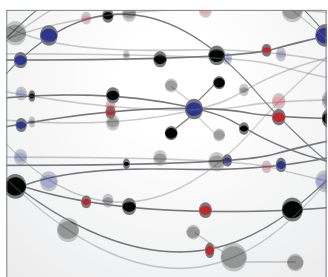

The Scientific World Journal
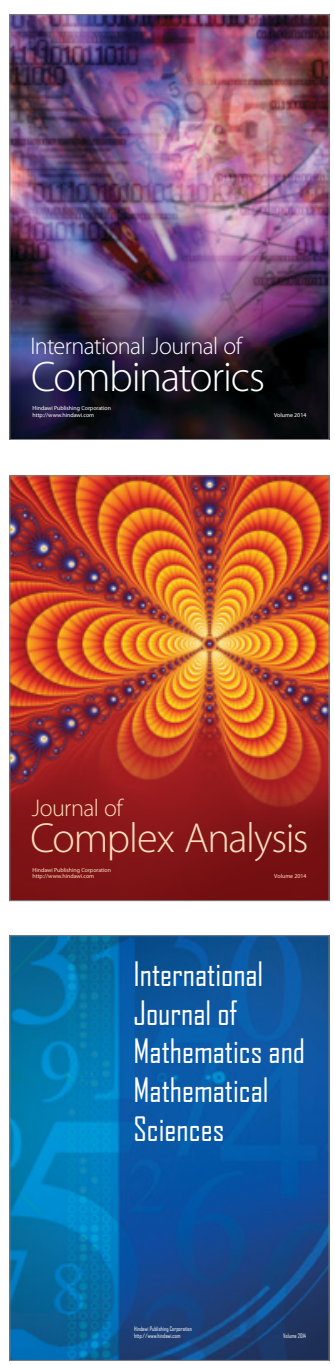
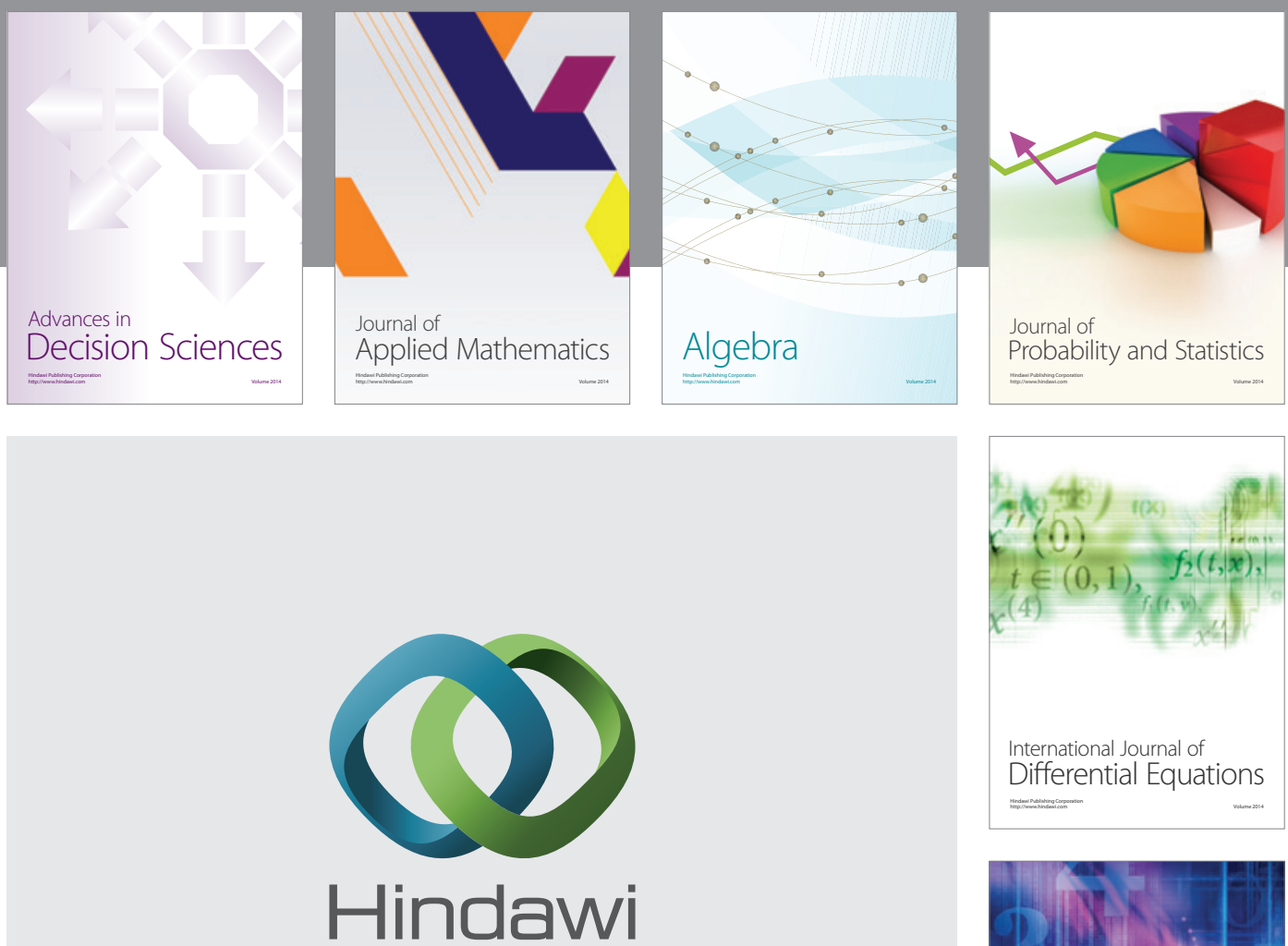

Submit your manuscripts at http://www.hindawi.com
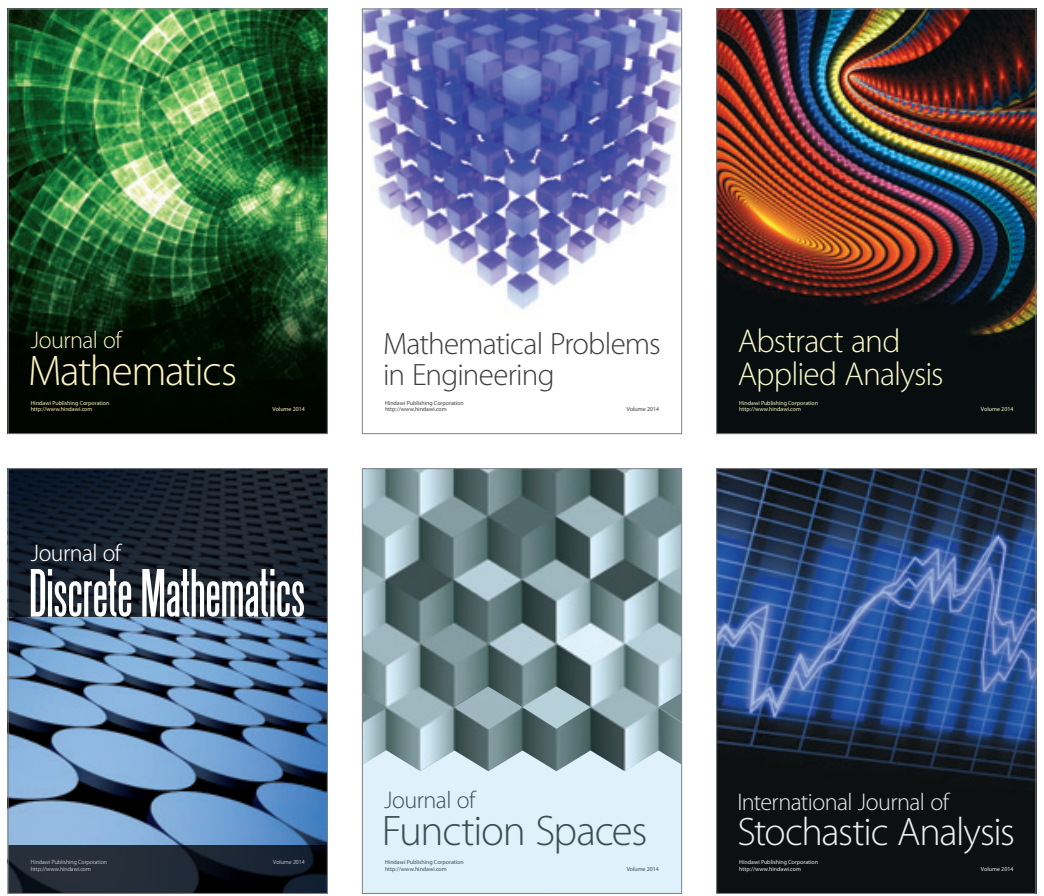

Journal of

Function Spaces

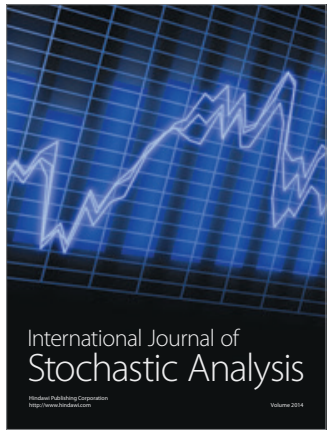

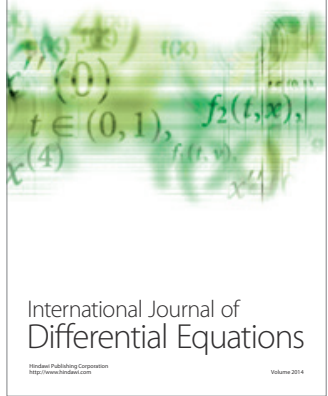
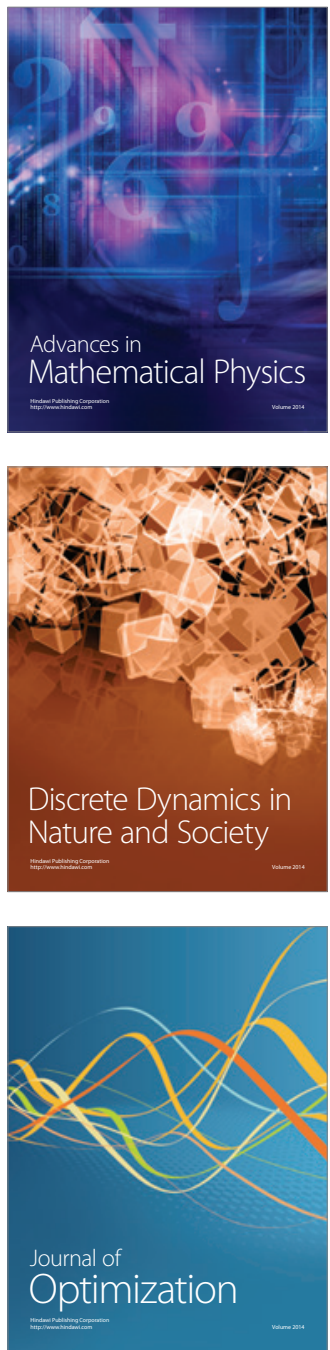